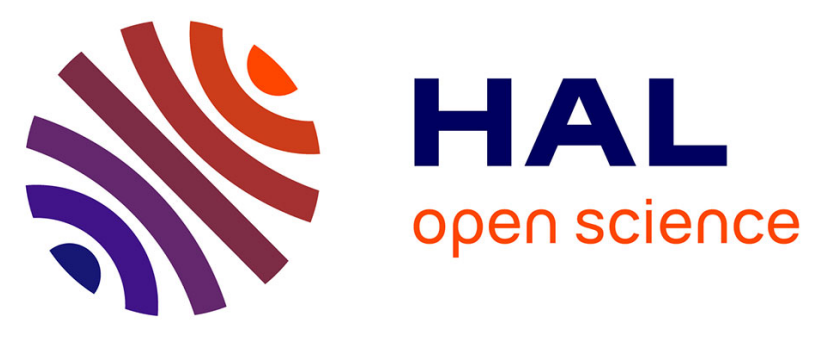

\title{
Low-Complexity Lattice Reduction Demapper for Massive Order One-Dimensional Non-Uniform Constellations
}

\author{
Jon Barrueco Gutierrez, Jon Montalban, Pablo Angueira, Charbel Abdel \\ Nour, Catherine Douillard
}

\section{To cite this version:}

Jon Barrueco Gutierrez, Jon Montalban, Pablo Angueira, Charbel Abdel Nour, Catherine Douillard. Low-Complexity Lattice Reduction Demapper for Massive Order One-Dimensional Non-Uniform Constellations. BMSB2018: IEEE International Symposium on Broadband Multimedia Systems and Broadcasting, Jun 2018, Valencia, Spain. 10.1109/BMSB.2018.8436872 hal-01814195

\author{
HAL Id: hal-01814195 \\ https://hal.science/hal-01814195
}

Submitted on 17 Feb 2020

HAL is a multi-disciplinary open access archive for the deposit and dissemination of scientific research documents, whether they are published or not. The documents may come from teaching and research institutions in France or abroad, or from public or private research centers.
L'archive ouverte pluridisciplinaire $\mathbf{H A L}$, est destinée au dépôt et à la diffusion de documents scientifiques de niveau recherche, publiés ou non, émanant des établissements d'enseignement et de recherche français ou étrangers, des laboratoires publics ou privés. 


\title{
Low-Complexity Lattice Reduction Demapper for Massive Order One-Dimensional Non- Uniform Constellations
}

\author{
J. Barrueco, Member, IEEE, J. Montalban, Member, IEEE, P. Angueira, Senior Member, IEEE, \\ C. Abdel Nour, Member, IEEE and C. Douillard, Member, IEEE
}

\begin{abstract}
This paper presents a low-complexity demapping method for demodulating M-ary one-dimensional non-uniform constellations. For uniform constellations, there are several algorithms for searching the closest point to the received observation. However, for non-uniform constellations these techniques are not valid. We propose a novel technique based on dividing the non-uniform constellation into lattices of identical dimensions. The value of the received symbol is modified in order to provide an entry to a look-up table where the closest point is stored. This solution provides low complexity for demodulation of the in-phase and quadrature PAMs of the nonuniform constellation. The complexity is $O\left(2+\log _{2} \sqrt{M}\right)$ while the exhaustive search is $O(\sqrt{M})$. The complexity implications in terms of memory and computations and the system performance are analyzed.
\end{abstract}

Index Terms - Soft detection, Non-Uniform Constellations, Lattice Reduction Demapper.

\section{INTRODUCTION}

$\mathrm{T}$ he necessity of providing higher system capacity with the same spectral resources for the new wireless communications systems results into the birth of advanced modulation schemes. Recently, non-uniform constellations (NUC) have been proposed [1]-[3] in order to increment the bit-interleaved coding and modulation (BICM) spectral efficiency [4], [5]. Up to now, the existing NUCs can be gathered into two families: one-dimensional (1D-NUC) and two-dimensional non-uniform constellations (2D-NUC). 2D-

This work was supported in part by the Basque Government (PREDOC Program and IT-683-13), in part by the University of the Basque Country (UFI 11/30) and in part by the Spanish Ministry of Economy and Competitiveness (project 5G-newBROS under Grant TEC2015-66153-P MINECO/FEDER, UE).

J. Barrueco, and P. Angueira are with the Department of Communications Engineering, University of the Basque Country, 48013 Bilbao, Spain (e-mail: jon.barrueco@ehu.eus; jon.montalban@ehu.eus; pablo.angueira@ehu.eus).

J. Montalban is with the Department of Electronic Technology, University of the Basque Country, 20018 Donostia, Spain (e-mail: jon.montalban@ehu.eus).

C. A. Nour and C. Douillard are with the Institut Mines-Télécom/Télécom Bretagne, CNRS Lab-STICC, UMR 3192, Brest 29238, France, and also with the Université Européenne de Bretagne, Brest, France (e-mail: firstname.lastname@imt-atlantique.fr).
NUCs provide slightly higher capacity gain than 1D-NUCs at the cost of higher demapping complexity. A solution for lowering this complexity for 2D-NUCs is proposed in [6], [7]. For massive order constellations (beyond 256 constellation points) the latency involved in 2D-NUCs make their use unfeasible in real time applications. For such high order constellations, 1D-NUCs are the most appropriate solution.

1D-NUCs have been included in the latest developed digital broadcasting systems, i.e., Digital Video Broadcasting - Next Generation Handheld (DVB-NGH) [8] and Advanced Television Systems Committee $3^{\text {rd }}$ Generation (ATSC 3.0) [9]. ATSC 3.0 includes 1D-1024NUCs providing system gains of up to $1.8 \mathrm{~dB}$ w.r.t. the gray labeled uniform QAM constellations with the same demapping complexity. The major restriction of massive order 1D-NUCs is the number of computations required in the demapping stage. Several methods based on decomposing the gray-labeled QAM into two independent in-phase (I) and quadrature (Q) gray-labeled PAMs are shown in [10], [11]. These proposals perform onedimensional soft demapping on the separated components of a received symbol. 1D-NUCs can be also decomposed into two independent non-uniform QAMs, reducing the number of Euclidean distances (ED) computed.

In hardware applications, the max-log approximation of the ML demapper is commonly used [12]. The number of mathematical computations is reduced w.r.t. ML but the number of EDs remains the same. The value of the loglikelihood ration (LLR) metric given by the max-log approximation is mainly influenced by the closest point to the received observation. The authors of this paper proposed in [13] a low-complexity demapper based on a simplified detection of the closest point to the received observation. Nevertheless, this solution is not valid for 1D-NUCs as the constellation points are not uniformly spaced. Another approach is considered in [14] for demapping non-uniform MPAM constellations efficiently based on finding the intervals where the LLRs are linear functions. The overall complexity is reduced from $\mathrm{O}(\sqrt{M})$ to $\mathrm{O}\left(\log _{2} \sqrt{M}\right)$.

In this paper, we take advantage of the decomposition of the 1D-NUCs into two non-uniform I/Q PAMs in order to design a simplified technique to detect the closest symbol to the 
received observation and compute the LLR bit metrics. New 1D-NUCs with negligible performance losses are designed in order to perform the proposed low complexity demapper. The overall complexity is $\mathrm{O}\left(2+\log _{2} \sqrt{M}\right)$ with negligible impact in memory requirements. Demapping complexity reduction of up to $89 \%$ for $1 \mathrm{D}-4096 \mathrm{NUC}$ with system performance losses below 0.3 and $0.5 \mathrm{~dB}$ compared to max-log and $\mathrm{ML}$ respectively are obtained with imperceptible increase in memory requirements. Results are shown for the ATSC 3.0 BICM system but can be applied to any wireless communications system.

The rest of the paper is organized as follows: Section II presents the proposed demapper. Section III shows the design of the new 1D-NUCs. Section IV explains the simplified detection of the closest point to the received observation. In Section $\mathrm{V}$ the complexity analysis and system performance of the demapper is carried out using the ATSC 3.0 BICM system. Finally, section VI gathers the main conclusions.

\section{PROPOSED DEMAPPER}

The max-log demapper is extensively used in hardware applications for digital demodulation. Max-log is an approximation of the ML providing lower number of mathematical operations. However, the number of EDs to calculate is the same in both demappers. The max-log computes all EDs between the received symbol and the constellation symbols and selects the minimum one. In BICM systems, the LLRs are calculated for each transmitted bit using the conditional probability of receiving $y$ given that the $b_{i}$ transmitted is $0 p\left(y \mid b_{i}\right.$ is 0$)$ and that $b_{i}$ transmitted is $1 p\left(y \mid b_{i}\right.$ is 1). For the max-log, the LLR value for bit $b_{i}$ is given by the two closest symbols to the received observation with bit $b_{i}=0$ and $b_{i}=1$, respectively. We propose a simplified detection of these two symbols based on dividing the 1D-NUC into lattices of the same dimension.

The minimum length of the lattices $d_{\min }$ is the minimum distance between adjacent constellation points of the designed 1D-NUC. The 1D-NUC is designed with the restriction that the distances between adjacent constellation symbols are multiples of $d_{\min }$. Then, the constellation diagram is divided into lattices of length $d_{\min }$. The length of the lattices is normalized to unity and the lattices are numbered from 1 to $n$, where $n$ is the number of lattices. Next, the received symbol is discretized taking values from 1 to $n+1$ corresponding to the boundaries of the lattices. The two closest symbols to the discrete received observation with bit $b_{i}=0$ and 1 are stored in a LUT. The closest symbols used for LLR calculation are found in the column specified by the value of the discrete received symbol.

\section{1D-NUC DESIGN}

The authors of this paper proposed in [1] the design of optimal NUCs for BICM systems. The procedure explained in [1] is followed in this work to design the 1D-NUCs. The distances between adjacent constellation points in these designed 1D-NUCs do not fulfill the requirements expressed

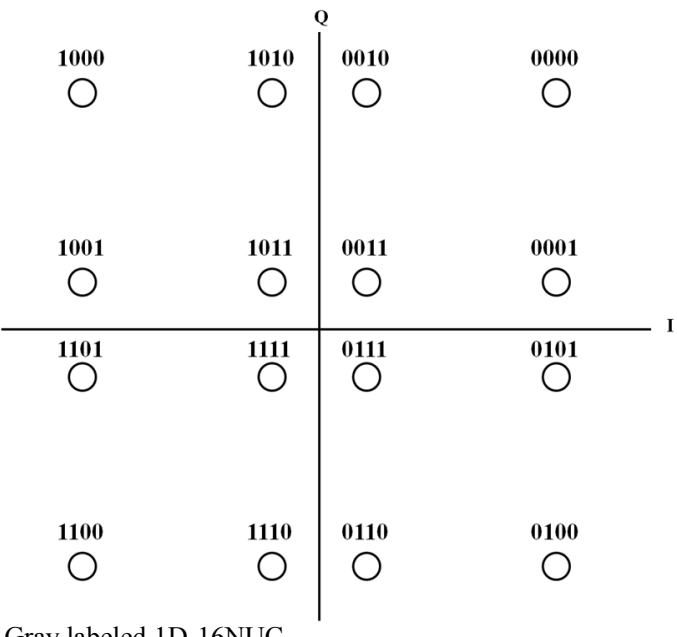

Fig. 1. Gray labeled 1D-16NUC

above.All the distances between adjacent points must be multiples of $d_{\min }$, and consequently, the designed 1D-NUCs are post-processed in order to fulfill the design condition.

Negligible losses are obtained for low and mid code rates due to the granularity given by the value of the $d_{\min }$. For this range of code rates (from $R=2 / 15$ to $R=10 / 15$ ), this is translated into negligible system performance losses. For high code rates, the BICM system capacity losses increase due to the low granularity of the value $d_{\text {min }}$. The system performance losses are below $0.2 \mathrm{~dB}$.

\section{Simplified Detection of the Closest Point to the RECEIVED OBSERVATION}

The objective is to create a LUT which stores the two closest symbols with $b_{i}=0$ and 1 respectively to a particular value of the received symbol. The value of the received symbol is modified representing the entry to the LUT and the LLR value using the max-log approximation is obtained. Three main steps are considered: divide the non-uniform PAM into uniform lattices, create the LUT and modify the value of the received observation. For simplicity, the gray labeled 1D16NUC shown in Fig. 1 is considered. We consider that the two non-uniform PAMs designed are similar with $\sqrt{M}$ points.

\section{1-) First step: Divide the non-uniform PAM into uniform} lattices

The key aspect of the first step is to create lattices of dimension unity whose limits in the constellation diagram are integers starting from 1. Each limit corresponds to one entry of the LUT. The processed value of the received observation is equal to one value of the boundaries. Figure 2 a shows one nonuniform PAM with four components $\left(S_{1}, S_{2}, S_{3}\right.$ and $\left.S_{4}\right)$ with multiple distances of $d_{\text {min }}$ between adjacent components. The value of $d_{\min }$ is 0.3922 . This non-uniform PAM has been designed following the procedure explained in Section III. Next, the positions of the PAM components are normalized dividing their positions by $d_{\min }\left(S_{1 b}, S_{2 b}, S_{3 b}\right.$ and $\left.S_{4 b}\right)$. This step is shown in Fig. 2b. Finally, the constellation points are shifted to the right of the constellation diagram, giving $\mathrm{S}_{1 \mathrm{c}}$ value 1 . In 


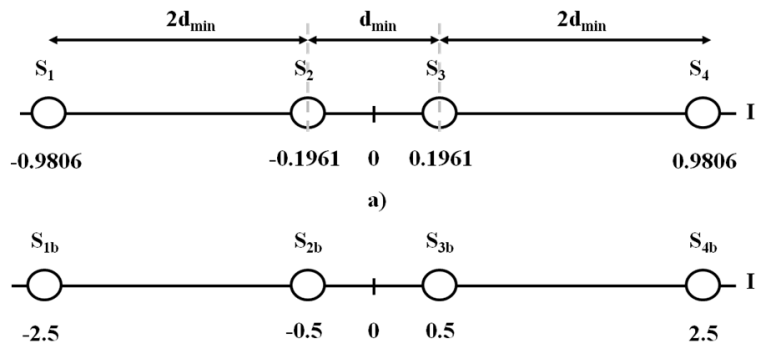

b)

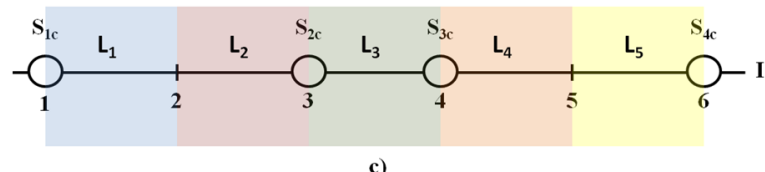

Fig. 2. Procedure of division of the non-uniform PAM into uniform lattices

this way, there are five different lattices (from $L_{1}$ to $L_{5}$ ) with a total of six boundaries (Figure 2c).

After processing the received observation (third step), the modified received constellation symbol can only take integer values, i.e., in this case from 1 to 6 .

\section{2-) Second step: Create the Look-Up Table}

The LUT is composed by $n$ rows and $m$ files, where $n$ is the number of bits of the PAM multiplied by two (as each bit can take value 0 or 1 ) and $m$ is the number of boundaries of the lattices.

$$
\begin{aligned}
& n=\log _{2} M \\
& m=L+1
\end{aligned}
$$

Eq. 1 represents the number of rows and columns of the LUT, where $M$ is the constellation order of the QAM constellation and $L$ is the number of unity lattices of the PAM.

Following the example, $M=16$ and $L=5$. Therefore the number of rows is $n=4$ and columns $m=6$. Each row represents each bit of the PAM constellation with value 0 or 1 . Each column includes the possible integer values of the boundaries. The I and Q PAMs of the designed 1D-16NUC contain $\frac{\log _{2} M}{2}$ bits each one, i.e., 2 bits. The in-phase PAM carries bit 2 and 4 of the 1D-16NUC and the quadrature PAM bit 1 and 3. Table I shows the LUT for the in-phase PAM. The content of the quadrature PAM is the same but for bits 1 and 3 .

3-) Third step: Modify the value of the received observation

The value of the received symbol is normalized by $d_{\min }$ and increased by the value of the highest constellation point, i.e., in the example $\mathrm{S}_{4 \mathrm{~b}}$. This value is increased by 1 , the same as in the first step, Fig. 2c for the PAM. Next, in order to obtain the closest boundary to the value of the received point, a value of 0.5 is added to the value of the received point (because the lattice has unity dimension) and the resulting value is rounded to the lowest integer value. Eq. 2 shows the calculation of the scaled value of the received symbol.
TABLE I

\begin{tabular}{|c|c|c|c|c|c|c|}
\hline \multirow{2}{*}{ Bit } & \multicolumn{6}{|c|}{ Scaled received observation } \\
\hline & 1 & 2 & 3 & 4 & 5 & 6 \\
\hline $\mathrm{b}_{2}=0$ & $\mathrm{~S}_{1 \mathrm{c}}$ & $\mathrm{S}_{1 \mathrm{c}}$ & $\mathrm{S}_{1 \mathrm{c}}$ & $\mathrm{S}_{4 \mathrm{c}}$ & $\mathrm{S}_{4 \mathrm{c}}$ & $\mathrm{S}_{4 \mathrm{c}}$ \\
\hline $\mathrm{b}_{2}=1$ & $\mathrm{~S}_{2 \mathrm{c}}$ & $\mathrm{S}_{2 \mathrm{c}}$ & $\mathrm{S}_{2 \mathrm{c}}$ & $\mathrm{S}_{3 \mathrm{c}}$ & $\mathrm{S}_{3 \mathrm{c}}$ & $\mathrm{S}_{3 \mathrm{c}}$ \\
\hline $\mathrm{b}_{4}=0$ & $\mathrm{~S}_{1 \mathrm{c}}$ & $\mathrm{S}_{1 \mathrm{c}}$ & $\mathrm{S}_{2 \mathrm{c}}$ & $\mathrm{S}_{2 \mathrm{c}}$ & $\mathrm{S}_{2 \mathrm{c}}$ & $\mathrm{S}_{2 \mathrm{c}}$ \\
\hline $\mathrm{b}_{4}=1$ & $\mathrm{~S}_{3 \mathrm{c}}$ & $\mathrm{S}_{3 \mathrm{c}}$ & $\overline{\mathrm{S}_{3 \mathrm{c}}}$ & $\mathrm{S}_{3 \mathrm{c}}$ & $\mathrm{S}_{4 \mathrm{c}}$ & $\mathrm{S}_{4 \mathrm{c}}$ \\
\hline
\end{tabular}

LOOK-UP TABLE EXAMPLE FOR 1D-16NUC

$$
r_{I-} \text { scaled }=\left\lfloor\frac{r_{I}+S_{\max }}{d_{\max }}+1.5\right\rfloor
$$

Where $r_{I}$ is the in-phase component of the received observation. $S_{\max }$ is the value of the highest constellation point and $r_{I}$ scaled is the value of the modified received point. It must be taken into account that $r_{\text {I_scaled }}$ could be positioned in the middle of two points which carry the same bit value. In this case, there exists a small deviation of the ED. However, as results in next section show, the impact in the system performance is negligible. The ED is easily calculated as shown in Equation 3.

$$
E D=\left|\frac{r_{I}+S_{\max }}{d_{\max }}+1.5-S_{x c}\right|
$$

Where $S_{x c}$ is the value obtained from the LUT.

\section{COMPLEXITy ANALYSIS AND SimUlation RESUlts}

\section{A. Complexity Analysis}

In this section we consider massive order 1D-NUCs, i.e., 1024 and 4096 constellation points. The main complexity of the demapper proposed comes from the Euclidean distances computations and the modification of the received observation. For each PAM, $1+\log _{2} \sqrt{M}$ distances are required and one step for the scaling of the received observation (with one division and three additions). The overall complexity is $\mathrm{O}\left(2+\log _{2} \sqrt{M}\right)$. The max-log method requires the computation of $\sqrt{M}$ distances between the received symbol and the constellation points. The overall complexity is $\mathrm{O}(\sqrt{M})$. If the proposed demapper is compared with the conventional methods, i.e., ML and max-log and 1D-1024NUC, 1D4096 NUCare considered, the complexity reduction is $78.1 \%$ and $87.5 \%$, respectively.

In terms of memory requirements, the total LUT size is ( $\mathrm{m} \mathrm{x}$ n) and the number of bits assigned to each cell is $\log _{2} \sqrt{M}$. Table II summarizes the memory requirements for the designed 1D-1024NUCs and 1D-4096NUCs in bytes. The memory is reduced by two as the LUT is the same for both I and Q components.

\section{B. System Performance Simulation Results}

All the results shown in this section have been obtained using an ATSC 3.0 BICM transceiver with 300 FEC blocks of 
TABLE II

LOOK-UP TABLE MEMORY REQUIREMENTS

\begin{tabular}{|c||c||c||}
\hline \multirow{2}{*}{ Code rate } & \multicolumn{2}{|c|}{ LUT memory requirements [Kilobytes] } \\
\cline { 2 - 3 } & 1D-1024NUC & 1D-4096NUC \\
\hline \hline $2 / 15$ & 0.01 & 0.25 \\
\hline \hline $3 / 15$ & 0.44 & 0.94 \\
\hline \hline $4 / 15$ & 0.55 & 1.93 \\
\hline \hline $5 / 15$ & 0.30 & 1.67 \\
\hline \hline $6 / 15$ & 0.67 & 0.75 \\
\hline \hline $7 / 15$ & 0.51 & 1.47 \\
\hline \hline $8 / 15$ & 1.44 & 2.76 \\
\hline \hline $9 / 15$ & 1.13 & 2.52 \\
\hline \hline $10 / 15$ & 1.00 & 0.87 \\
\hline \hline $11 / 15$ & 0.14 & 0.63 \\
\hline \hline $12 / 15$ & 0.12 & 0.33 \\
\hline \hline $13 / 15$ & 0.1 & 0.29 \\
\hline
\end{tabular}

length 64,800 bits [15]. Low density parity check (LDPC) code options $(R=2 / 15$ to $R=13 / 15)$ included in ATSC 3.0 [16], with 1D-1024NUC and 1D-4096NUCs under Rayleigh independent and identically distributed (iid) channel are considered. Results for the ML and max-log are obtained using the 1D-NUCs defined in ATSC 3.0. For the proposed demapper, newly designed 1D-NUCs explained in Section III are used.

Table III shows the system performance losses in $\mathrm{dB}$ of the proposed solution (newly designed 1D-NUCs and low complexity demapper) compared with $\mathrm{ML}$ and max-log detection with ATSC 3.0 1D-NUCs. The system performance losses of the designed demapper against the ML range from 0.1 to $0.4 \mathrm{~dB}$ for $1 \mathrm{D}-1024 \mathrm{NUCs}$ and from 0.1 to $0.5 \mathrm{~dB}$ for 1D-4096NUCs. As it is shown in [7] and [14] the max-log approximation has a perceptible deviation from the value given by the ML for non-uniform constellations. When the constellation points are not equidistant, the ML cannot be purely approximated by the max-log. Results in Table III show that the system performance losses of the proposed solution compared with the max-log are negligible for 1D-4096NUCs (maximum of $0.1 \mathrm{~dB}$ for high code rates). In the case of 1D1024NUCs, losses of up to $0.3 \mathrm{~dB}$ are present for high code rates. For mid and low code rates, the performance losses are negligible. The system performance losses w.r.t. the max-log come from the restriction imposed in the design of 1D-NUCs.

\section{CONCLUSIONS}

In this paper, a low complexity demapper for massive order 1D-NUCs has been proposed. It is based on finding the closest constellation points to the received observation in an efficient way. The 1D-NUC is divided into two PAMs. The constellation points of the PAMs are transformed in order to create equidistant lattices. The boundaries of the lattices are used to create a LUT with the closest symbols depending of the value of the scaled received observation.
TABLE III

SySTEM PERFormance LOSSES OF THE PROPOSED DEMAPPER COMPARED WITH THE ML AND MAX-LOG DEMAPPERS

\begin{tabular}{|c|c|c|c|c|}
\hline \multirow{3}{*}{$\begin{array}{c}\text { Code } \\
\text { rate }\end{array}$} & \multicolumn{4}{|c|}{ Performance Losses [dB] } \\
\hline & \multicolumn{2}{|c|}{ 1D-1024NUC } & \multicolumn{2}{|c|}{ 1D-4096NUC } \\
\hline & $\mathrm{ML}$ & Max-Log & $\mathrm{ML}$ & Max-Log \\
\hline $2 / 15$ & 0.1 & 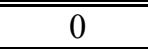 & 0.5 & 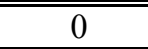 \\
\hline $3 / 15$ & 0.3 & 0 & 0.4 & 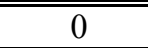 \\
\hline $4 / 15$ & 0.3 & $\overline{0}$ & 0.4 & $\overline{0}$ \\
\hline $5 / 15$ & 0.4 & 0 & 0.2 & 0 \\
\hline $6 / 15$ & 0.2 & 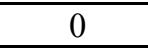 & 0.2 & 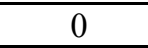 \\
\hline $7 / 15$ & 0.2 & 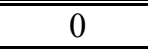 & 0.1 & 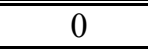 \\
\hline $8 / 15$ & 0.1 & 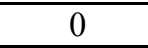 & 0.2 & 0 \\
\hline 9/15 & 0.1 & 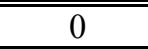 & 0.2 & 0 \\
\hline $10 / 15$ & 0.1 & 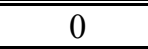 & 0.1 & 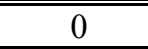 \\
\hline $11 / 15$ & 0.3 & 0.2 & 0.2 & 0 \\
\hline $12 / 15$ & 0.2 & 0.2 & 0.2 & 0.1 \\
\hline $13 / 15$ & 0.3 & 0.3 & 0.3 & 0.1 \\
\hline
\end{tabular}

The complexity of this solution for each PAM is $\mathrm{O}\left(2+\log _{2} \sqrt{M}\right)$ as opposed to $\mathrm{O}(\sqrt{M})$ for the ML and max$\log$ demappers where $M$ is the constellation order of the M-ary 1D-NUC. Negligible system performance losses are obtained for low and mid code rates. For high code rates, the performance losses are below 0.3 and $0.1 \mathrm{~dB}$ for $1 \mathrm{D}-1024 \mathrm{NUC}$ and 1D-4096NUC, respectively.

\section{REFERENCES}

[1] J. Barrueco et al., "Constellation Design for Bit-interleaved Coded Modulation (BICM) Systems in Advanced Broadcast Standards," IEEE Trans. Broadcast., DOI: 10.1109/TBC.2017.2677259.

[2] J. Barrueco et al., "Combining advanced constellations and SSD techniques for optimal BICM capacity," in Proc. IEEE Int. Symp. Broadband Multimedia Syst. Broadcast. (BMSB), Ghent, Belgium, pp. 14, Jun. 2015.

[3] J. Zoellner and N. Loghin, "Optimization of high-order non-uniform QAM constellations," in Proc. Int. Symp. Broadband Multimedia Syst. Broadcast., London, U.K., Jun. 2013, pp. 1-6.

[4] G. Caire, G. Taricco, and E. Biglieri, "Bit-interleaved coded modulation," IEEE Trans. Inf. Theory, vol. 44, no. 3, pp. 927-946, May 1998.

[5] G. Caire, G. Taricco, and E. Biglieri, "Capacity of bit-interleaved channels,"IEEE Electron. Lett., vol. 32, no. 12, pp. 1060-1061, Jun. 1996.

[6] M. Fuentes, D. Vargas, and D. Gómez-Barquero, "Low-Complexity Demapping Algorithm for Two-Dimensional Non-Uniform Constellations," IEEE Trans. Broadcast., vol. 62, no. 2, pp. 375-383, June 2016

[7] C. Barjau, M. Fuentes, T. Shitomi, and D. Gomez-Barquero, "MIMO Sphere Decoder with Successive Interference Cancellation for TwoDimensional Non-Uniform Constellations," IEEE Communications Letters, DOI: 10.1109/LCOMM.2017.2653775.

[8] Digital Video Broadcasting, Next Generation Broadcasting System to Handheld, Physical Layer Specification (DVB-NGH), DVB Doc. A160, Nov. 2012.

[9] ATSC Proposed Standard: Physical Layer Protocol (A/322), Doc. S32230r56, Adv. Telev. Syst. Comm., Washington, DC, USA, June 2016. 
[10] F. Tosato, and P. Bisaglia, "Simplified soft-output demapper for binary interleaved COFDM with application to HIPERLAN/2," IEEE International Conference on Communications, New York, U.S.A., Aug. 2012.

[11] Q. Wang, Q. Xie, Z. Wang, S. Chen, and L. Hanzo, "A Universal LowComplexity Symbol-to-Bit Soft Demapper," IEEE Trans. On Vehicular Technology., vol. 63, no. 1, pp. 119-130, Aug. 2013.

[12] R. Shrestha, and R. Paily, "Hardware Implementation of Max-Log-MAP algorithm based on MacLaurin series for turbo decoder," International Conference on Communications and Signal Processing, Calicut, India, Feb. 2011.

[13] A. Haroun, C. Abdel Nour, M. Arzel, and C. Jego, "Low-Complexity Soft Detection of QAM Demapper for a MIMO System," IEEE Communications Letters, vol. 20, no. 4, pp. 732-735, Feb. 2016.

[14] M. Sandell, F. Tosato, and A. Ismail, "Low-Complexity Max-log LLR Computation for Nonuniform PAM Constellations," IEEE Communications Letters, vol. 20, no. 5, pp. 838-841, May 2016.

[15] L. Michael and D. Gomez-Barquero, "Bit-interleaved coded modulation (BICM) for ATSC 3.0," IEEE Trans. Broadcast., vol. 62, no. 1, pp. 181188, Mar. 2016.

[16] K.-J. Kim et al., "Low-density parity-check codes for ATSC 3.0," IEEE Trans. Broadcast., vol. 62, no. 1, pp. 189-196, Mar. 2016. 